\title{
Application of gold-labeled antibody biosensor in simultaneous determination of total aflatoxins using artificial neural network
}

\author{
Asiye Saidi · Mohammad Mirzaei
}

Received: 19 January 2013/Accepted: 9 July 2013/Published online: 23 July 2013

(C) The Author(s) 2013. This article is published with open access at Springerlink.com

\begin{abstract}
Preparation of antibody-coated gold nanoparticles (GNPs) specific to aflatoxins B1, B2, G1 and G2 and its use in developing aflatoxins diagnostic method were presented in this paper. The formation of gold-labeled antibodies was accomplished at optimal condition. Due to severe overlapping between the emission profiles for the aflatoxins, they cannot be determined by direct inspection of data. The strategy used in this study, constituted by artificial neural network (ANN), was easy to implement and to originate reliable results. ANN can be successfully applied to spectrofluorimetric spectra matrices to simultaneous determination of total aflatoxins. Quantitative results obtained using ANN method for aflatoxins in pistachio nuts samples were compared to those obtained using the HPLC method. Obtained results using these two methods did not show significant differences.
\end{abstract}

Keywords Aflatoxins · Gold nanoparticle · Antibody · Artificial neural network

\section{Introduction}

Mycotoxins are secondary metabolites produced by fungi which frequently contaminate plant products worldwide. Aflatoxins are among the most toxic mycotoxins [1-3]. These toxic compounds are potent carcinogenic and mutagenic secondary metabolites produced by the Aspergillus genus, especially A. flavus and A. parasiticus [4, 5]. Therefore, the contamination of food products such as

\footnotetext{
A. Saidi · M. Mirzaei ( $)$

Department of Chemistry, Shahid Bahonar University

of Kerman, 22 Bahman Boulevard, 76169 Kerman, Iran

e-mail: m_mirzaei@mail.uk.ac.ir
}

cereals, nuts and the other commodities with these mycotoxins is controlled by legal limits (as maximum tolerated level, MTL) [6]. Pistachio nut is one of the food commodity classes with the highest risk of aflatoxins contamination [6], with Iran as a major worldwide pistachio producer. Institute of Standards and Industrial Research of Iran (ISIRI) has set a MTL of 5 and $15 \mu \mathrm{g} \mathrm{Kg}^{-1}$ for AfB1 and total aflatoxins, respectively, in 2002. Among 20 types of aflatoxins, only aflatoxins B1, B2, G1 and G2 play a vital role in foods and feeds [7, 8]. The simultaneous identification of total aflatoxins in a single test considerably reduces the time and costs of each analysis and is the most attractive approach practically. Currently, many simultaneous methods, such as high-performance liquid chromatography (HPLC), liquid chromatography-mass spectrometry (LC-MS) and magneto resistive-based immunoassay and also, several immunological methods such as enzyme-linked immunosorbent assay and fluorescence polarization immunoassay have been developed for the detection and identification of aflatoxins in food and feedstuffs [9-14]. However, most of these methods are time consuming, costly, laborious, and require expensive instruments. The measurement of antibody or antigen concentrations based on biospecific recognition interactions such as biosensors has been considered as a major analytical method and used in environmental and biochemical studies. This method has generated much interest due to its cost-effectiveness, sensitivity and specificity [1519]. Gold nanoparticles (GNPs) have recently attracted significant attention due to their non-toxic nature and excellent biological compatibility $[20,21]$. Therefore, gold-labeled antibodies have provided attractive means for developing biosensors without the handling of toxic reagents [22-25]. Unlike fluorescence or enzyme-detection systems, gold-labeled antibodies are more stable and easy 
to use. There are no needs for fussy operations as incubation, washing and enzymatic reactions during signal generation. Furthermore, nanoscale surfaces provided by GNPs could accelerate antibody-antigen reaction sufficiently, which supply an amplified signal [26]. One of the best detection techniques is fluorescence, which has achieved major developments in bioanalytical applications due to its wonderful sensitivity and selectivity. Aflatoxins compounds can be determined by molecular fluorescence, but the fluorescence spectra of them severely overlapped. However, in complex mixtures, spectral overlapping is often a serious problem and separation techniques must be used after spectrofluorimetric techniques. In order to perform a global analysis on the spectral overlapping of total aflatoxins and simultaneous determination of them, chemometric methods must be employed. Recently, chemometric methods such as principal component regression (PCR), partial least square (PLS) and artificial neural network (ANN) have found increasing applications for multicomponent determinations [27-30]. The ANN is an emerging non-linear computational modeling method that is used in foodstuff analysis recently [31]. This is most probably due to its properties of non-linearity, input-output mapping, compatibility, and neurobiological analogy [32]. The use of neural networks in chemometrics has increased during the last decades [33, 34]. It has been demonstrated that it is possible to obtain excellent results in multivariate calibration problems using ANN. This method allowed for the rapid determination of aflatoxins by spectrofluorimetric procedures without requiring the prior knowledge of the involved analytical systems. This paper describes the application of ANN method to set spectrofluorimetric data from aflatoxins gold-labeled antibody complex. The analysis of the spectrofluorimetric data by ANN allows the simultaneous determination of the concentration of aflatoxins present in the samples with several advantages, such as procedure simplicity, rapid operation and immediate results, low cost, and no requirement for skilled technicians or expensive equipment. The accuracy and the precision of the method were established by the analysis of spiked samples. Validation of the developed method was accomplished by HPLC analysis of different samples.

\section{Experimental}

\section{Materials}

Standard solutions contain mixture of aflatoxins B1, G1 $\left(1,000 \mu \mathrm{g} \mathrm{Kg}^{-1}\right)$ and $\mathrm{B} 2, \mathrm{G} 2\left(200 \mu \mathrm{g} \mathrm{Kg}^{-1}\right)$ in methanol were purchased monthly from Marjaan Khatam (Training, Research \& Q.C. Lab. Services, Tehran, Iran). These solutions were stored at $-18{ }^{\circ} \mathrm{C}$. All needed working solutions were prepared daily by diluting these standard solutions. Anti-aflatoxin (B1, B2, G1 and G2) mouse monoclonal antibody, hydrogen tetrachloroaurate (III) $\left(\mathrm{HAuCl}_{4} \cdot 3 \mathrm{H}_{2} \mathrm{O}\right)$ and sodium citrate were obtained from Sigma-Aldrich (USA) and used without further purification. Phosphate-buffered saline (PBS, pH 7.4, 0.01 M in $0.85 \% \mathrm{NaCl}$ ) was prepared. All other chemicals were of analytical grade and were used without further purification.

Instruments and software

UV-Vis absorption spectra were carried out on a Spectrophotometer (VARIAN Cary 50). The transmission electron microscopy (TEM) images were taken with a Philips CM-10 instrument. All the fluorescence measurements were performed on an RF-5301PC spectrofluorimeter (Shimadzu Corporation, Japan). HPLC analyses were performed with a Waters 2690 combined with a RF10AXL fluorescence detector. Several programs implemented in MATLAB, obtained, were used to perform ANN modeling. The SPSS version 10.0 software was used for the statistical treatment of the data.

\section{Synthesis and characterization of GNPs}

An aqueous solution of chloroauric acid $(50 \mathrm{~mL}$ of $0.01 \%$ $[\mathrm{w} / \mathrm{v}] \mathrm{HAuCl}_{4} \cdot 3 \mathrm{H}_{2} \mathrm{O}$ ) was heated to the boiling point, and $2 \mathrm{ml}$ of $1 \%$ sodium citrate was added rapidly with constant stirring. The color of the solution changed from yellow purple to red within $1 \mathrm{~min}$. The solution was allowed to boil for another $10 \mathrm{~min}$. After cooling, in dark, the solution volume was made up to $50 \mathrm{~mL}$ with distilled water. The colloidal solution was stored in a dark bottle at $4{ }^{\circ} \mathrm{C}$ and was used in the preparation of the gold-labeled antibody. The size and shape of the synthesized GNPs were characterized by TEM.

Formation of gold-labeled antibody

Monoclonal antibody $\left(1 \mathrm{mg} \mathrm{L}^{-1}, 1 \mathrm{~mL}\right)$ prepared in $\mathrm{pH} 7.4$ phosphate-buffered solution $(0.01 \mathrm{M})$ was added to $1 \mathrm{~mL}$ of colloidal gold solution while stirring. The $\mathrm{pH}$ of the GNP solution was adjusted to $\mathrm{pH} 7.4$ by addition of dilute $0.01 \mathrm{M} \mathrm{Na}_{2} \mathrm{CO}_{3}$ before adding the antibody. The solution was stored for a period of $1.5 \mathrm{~h}$ at room temperature and centrifuged $\left(5,000 \mathrm{rpm}\right.$ at $\left.4{ }^{\circ} \mathrm{C}\right)$ in an Eppendorf centrifuge (Model 5804R, Germany) for $30 \mathrm{~min}$ to remove unconjugated antibody from the solution. The obtained pellet was dispersed in $2 \mathrm{~mL}$ PBS at $\mathrm{pH} 7.4$ and stored at $4{ }^{\circ} \mathrm{C}$ for further experiments. The formation of gold-labeled antibodies was monitored by UV-Visible light measurements, TEM and fluorescence spectroscopy. 
Optimal condition studies for conjugation between gold nanoparticle and antibody

For conjugation, antibody was directly adsorbed on the GNP surfaces, mediated mainly by London-van der waals force and hydrophobic interaction [35]. The colloidal gold was formed in solution by virtue of a balance between electrostatic repulsion and London-van der Waals attraction among the particles. However, on addition of ionic substance, the attracting force becomes greater than the counteraction, which leads to an aggregation accompanying a color change from red $\left(\lambda_{\max } \sim 520 \mathrm{~nm}, \mathrm{~A}_{520}\right)$ to blue $\left(\lambda_{\max } \sim 580 \mathrm{~nm}, \mathrm{~A}_{580}\right)$ [35]. Coating the colloidal surfaces with protein molecules, such as antibody, can prevent this instability. GNPs in the colloidal and stable state have the $\lambda_{\text {max }}$ at $520 \mathrm{~nm}$, but in the vicinity at the other ions, the particles showed red shifted and the color of the solution changes to blue. This phenomenon is indicative that the nanoparticles are in coagulum state. Therefore, optimal conditions of $\mathrm{pH}$ and antibody concentration for the coating can be determined by comparing the absorption between $\lambda_{\max } 520 \mathrm{~nm}$ and $\lambda_{\max } 580 \mathrm{~nm}$. GNP suspension adjusted to $\mathrm{pH}$ range of 5-9 was pipetted into a series of tubes. Antibody solution $(0.2-2 \mathrm{mg} / \mathrm{L}, 1 \mathrm{~mL})$ was added to each colloidal gold solution diluted in a series of concentrations. Each tube received $1 \mathrm{~mL}$ of $10 \% \mathrm{NaCl}$ and was shaken for $5 \mathrm{~min}$. Absorption of each tube at 520 and $580 \mathrm{~nm}$ was determined $10 \mathrm{~min}$ later.

\section{Preparation of the pistachio samples}

The ground pistachio kernels (100 g) were mixed with distilled water $(150 \mathrm{~mL})$ and grinded. For every $50 \mathrm{~g}$ portion of grinded sample, $5 \mathrm{~g} \mathrm{NaCl}$ and $220 \mathrm{~mL}$ methanol: $n$-hexane (volumes 120:100 mL) were added into $500 \mathrm{~mL}$ Erlenmeyer flask. The salt probably increases the ionic strength of the solvent that improves the extraction selectivity and the yield of the aflatoxins extraction process. The mixture agitated intensively on a stirrer (Heidolph, Germany) for $3 \mathrm{~min}$. The extract was filtered through Whatman No. 1 filter paper. The mixture of extraction solvent and $n$ hexane was collected at the system exit within two phases. The fat of the pistachio samples was extracted by $n$-hexane (upper phase) and it was separated and discarded. Aflatoxins were extracted by the solution of $100 \%(\mathrm{v} / \mathrm{v})$ methanol (lower phase). Then $20 \mathrm{~mL}$ of extracted solvent was diluted with $130 \mathrm{~mL}$ of distilled water.

Analysis of aflatoxins

Solutions containing a constant concentration of goldlabeled antibodies equal to $0.05 \mathrm{mg} \mathrm{L}^{-1}$ and a variable concentration of four aflatoxins have been prepared. The range of concentration of the total aflatoxins was about $0-54 \mu \mathrm{g} \mathrm{Kg}^{-1}$. The solutions were centrifuged (5,000 rpm at $4{ }^{\circ} \mathrm{C}$ ) for $30 \mathrm{~min}$. The resultant pellets were resuspended in $2 \mathrm{~mL}$ of the methanol-water solution $(40: 60, \mathrm{v} / \mathrm{v})$. For each measurement, the final solutions were transferred into a spectrofluorimetric cell to record the fluorescence versus wavelength. The selection of the optimum excitation wavelength for recording fluorescence spectra was examined and finally excitation wavelength was set to $365 \mathrm{~nm}$, and emission wavelengths were set from 400 to $600 \mathrm{~nm}$ [36]. Emission wavelength increments were $1 \mathrm{~nm}$ with a slit of $10 \mathrm{~mm}$ and using $1 \mathrm{~cm}$ path length quartz cell. Spectrofluorimetric data were analyzed by ANN method.

\section{Artificial neural network}

Artificial neural networks (ANNs) are mathematical systems that simulate biological neural networks. They are greatly distributed interconnections of compatible nonlinear processing elements or neurons, in which it resembles the human brain in two aspects, i.e., learning process is needed for the network to acquire knowledge from its environment, and inter neuron connection strength or synaptic weights are used to store the acquired knowledge [32]. These properties of ANN provide higher flexibility and capability in data fitting, prediction, and modeling of non-linear relationships. A detailed description of the theory behind a neural network has been adequately described elsewhere [37-40]. The structure of the network comprised of three node layers: an input, a hidden and an output layer. The input nodes transferred the weighted input signal to the nodes in the hidden layer, and the same as the hidden nodes for the output layer. A connection between the nodes of different layers was represented by a weight $\left(w_{j i}\right)$. During the training process, the correction of weight $\left(\Delta w_{j i}\right)$ was defined as follows:

$\Delta w_{j i}(n+1)=\eta \delta_{j} o_{i}+\alpha \Delta w_{j i}(n)$

where $\delta_{j}$ is the error term, $o_{i}$ is the output of node $j, \eta$ is the learning rate, $\alpha$ is the momentum and $n$ is the iteration number. The iteration would be finished when the error of prediction reached a minimum. A non-linear transformation, a sigmoidal function, was applied between the input and output of each node.

The most popular method for data compression in chemometrics is principal component analysis (PCA) [41]. PCA is appropriate, when we have obtained measures on a number of observed variables and wish to develop a smaller number of artificial variables that will account for most of the variance in the observed variables. PCA became an ideal tool to remove possible complications caused by multicollinearity from the independent variables. The main advantage of PCA is that it compresses the data 
by reducing the number of dimensions, without much loss of information. In the course of performing a PCA, it is possible to calculate a score for each subject on a given PC. Although a large number of components may be extracted in this way, only the first few components will be important enough to be retained for interpretation. The first component extracted in a PCA accounts for a maximal amount of total variance in the observed variables. The second component extracted will account for a maximal amount of variance in the data set that was not accounted by the first component. Reducing the number of inputs to a network reduces training time and, is therefore, a favorable objective as it allows more network design to be evaluated in a given time. In practice, principle components (PCs) are often successfully used as inputs in ANN instead of original data.

In training process, the network weights, the number of nodes in the hidden layer, number of epoch in the beginning of over-fitting and the kind of training algorithm were optimized. In order to optimize the number of nodes in the hidden layer and to control over-fitting of the network, mean square error (MSE) of training and prediction sets were monitored during the training procedure. MSE provides a good index for the average error of different ANN models. It was defined by Eq. 2 .

$\operatorname{MSE}=1 / n \sum\left(y_{c j}-y_{e j}\right)^{2}$

where $n$ represents the number of samples, and $y_{c j}$ and $y_{e j}$ represent the values of computed and experimental output of the jth sample, respectively.

Determination of aflatoxins by the HPLC method

HPLC was used as reference method for the determination of aflatoxins in pistachio nuts [42]. Aflatoxins were isocratically separated using a HPLC (Waters model 2690), with a C-18 column $(200 \times 4.6 \mathrm{~mm})$, a fluorescence detector and $10 \mu \mathrm{L}$ of sample injection. The mobile phase was methanol-water $(40: 60, \mathrm{v} / \mathrm{v})$ at a flow rate of $1 \mathrm{~mL} \min ^{-1}$. The fluorescence detector was set at the excitation wavelength of $365 \mathrm{~nm}$ and emission wavelength of $450 \mathrm{~nm}$. Quantification of each toxin was performed by measuring their peak areas and comparing them with their relevant standard calibration curve.

\section{Results and discussion}

Characterization of gold-labeled antibody conjugates

With the consideration of optimal conditions of antibody and colloidal gold conjugation, the minimal concentration of antibody to stabilize colloidal gold was approximately $1 \mathrm{mg} \mathrm{L}^{-1}$ (Fig. 1a), and the $\mathrm{pH}$ of the gold-labeled antibody solution was determined to be 7.4 (Fig. 1b). Figure 2 shows the UV-Vis spectra of the colloidal gold and conjugates, prepared as described previously. Included are the spectra of the GNP solution (curve a) and gold-labeled antibody conjugate at $\mathrm{pH} 7.4$ (curve b). A peak at $\sim 519 \mathrm{~nm}$ in curve (a) is due to the surface plasmon resonance of GNPs. After addition of the antibody, the surface plasmon band broadened and red shifted due to interaction of the antibody with colloidal gold particles.

Figure 3 shows the TEM images of the GNPs and the gold-labeled antibodies formed on a carbon-coated copper grid. TEM images indicate that the gold colloids are in monodispersional with a narrow diameter distribution. The analysis shows that the particles formed were spherical and the average diameter of GNPs was about $3 \mathrm{~nm}$.

The immunoreactivity of antibody depends upon the tertiary structure of the antibody remaining unperturbed after formation of conjugates with GNP [43]. The tertiary structure of the antibody can be studied by fluorescence measurements, by exciting the sample at a particular wavelength and monitoring the fluorescence emission from the tryptophan or tyrosine residues in the antibody. Figure 4 (curve a) shows the fluorescence spectrum of free antibody ( $\mathrm{pH} 7.4,0.01 \mathrm{M}$ PBS). The sample was excited at $275 \mathrm{~nm}$, and the emission was monitored in the range $300-400 \mathrm{~nm}$. A broad band was observed at $336 \mathrm{~nm}$ and indicates intactness of the tertiary structure of antibody in solution. The curve of conjugate showed the fluorescence
Fig. 1 Light absorption of conjugate at a different antibody concentrations $(0.2-2 \mathrm{mg} / \mathrm{L})$ and $\mathbf{b}$ different $\mathrm{pH}$ (5-9)
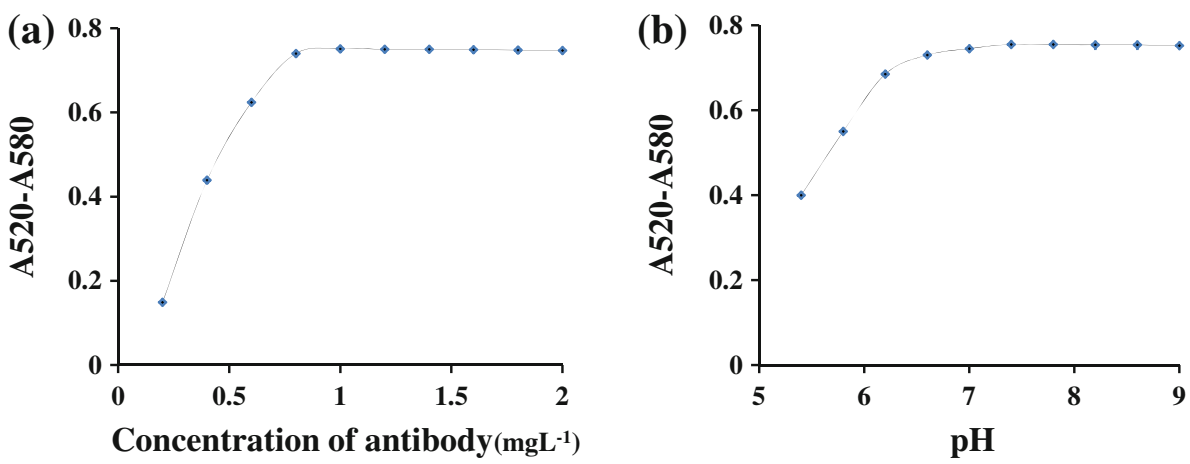


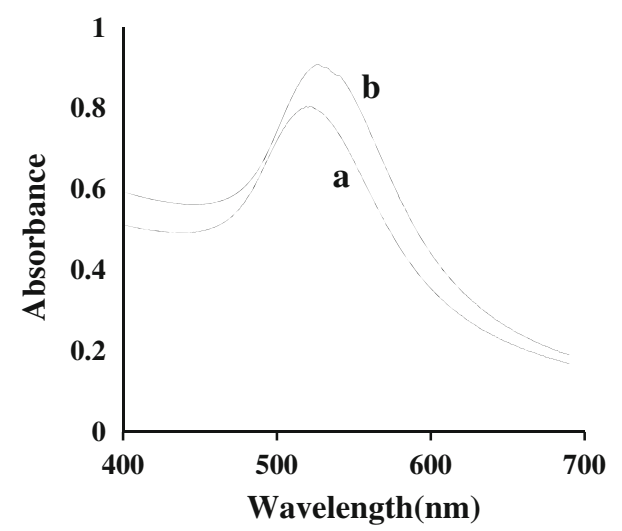

Fig. 2 UV-Visible spectra of GNPs ( $a$ ) and gold-labeled antibodies (b)

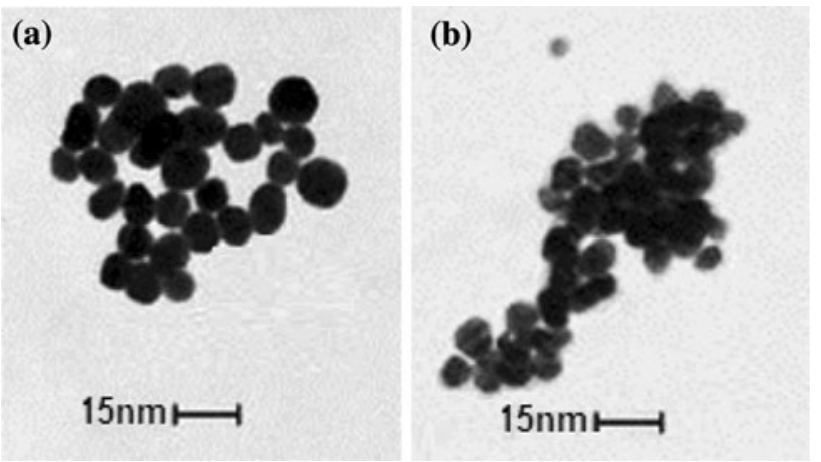

Fig. 3 TEM images of GNPs (a) and gold-labeled antibodies (b)

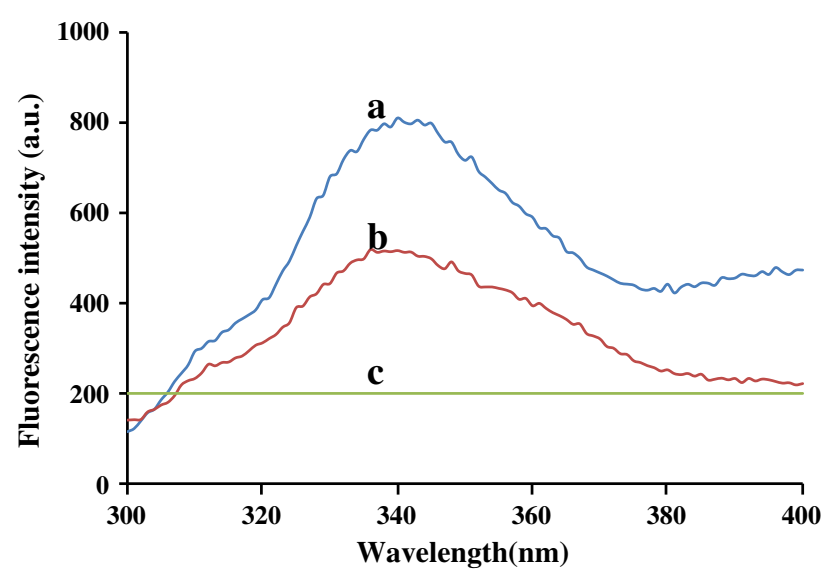

Fig. 4 Fluorescence spectra of free antibodies (a), gold-labeled antibodies $(b)$, decanted solution of gold-labeled antibodies after centrifuge $(c)$

spectrum recorded under the same emission conditions (curve b). The nature of the curve and peak position was quite similar to the free antibody, indicating the intactness of the antibody after the conjugation. Comparing the tryptophan emission intensities of the conjugate versus that of the free antibody, a significant amount of fluorescence quenching of tryptophan residues in the antibody was observed. Gold-labeled antibodies were centrifuged. After separation of pellet from clear phase of solution, the fluorescence of clear solution was recorded. As shown in Fig. 4 (carve c), clear solution was without any antibodies. This indicates that reaction between antibody and GNPs was complete.

\section{Multivariate calibration with ANN}

In this work, data sets coming from fluorescence intensity of samples consist of total aflatoxins. As shown in Fig. 5, the fluorescence spectra of them are severely overlapped. For simultaneous determination of total aflatoxins, the spectrofluorimetric data obtained from experiments were processed by ANN, which was trained with the backpropagation of errors learning algorithm. The reduced spectrofluorimetric data with PCA were used as the input of ANN. A three-layer ANN with a sigmoid transfer function was considered as primary architecture of the network. Figure 6 shows the plots of MSE of training and test as a function of the number of epochs for aflatoxins components. The minimum MSEs to control over-fitting appear after 8 epochs. As can be seen from Fig. 6, after 8

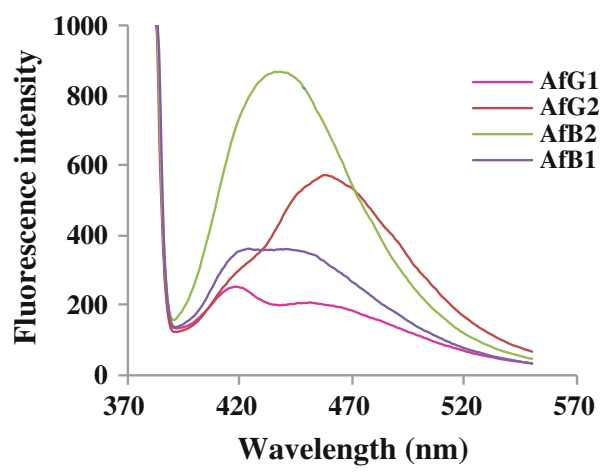

Fig. 5 Fluorescence emission spectra of pure aflatoxins B1, B2, G1, G2

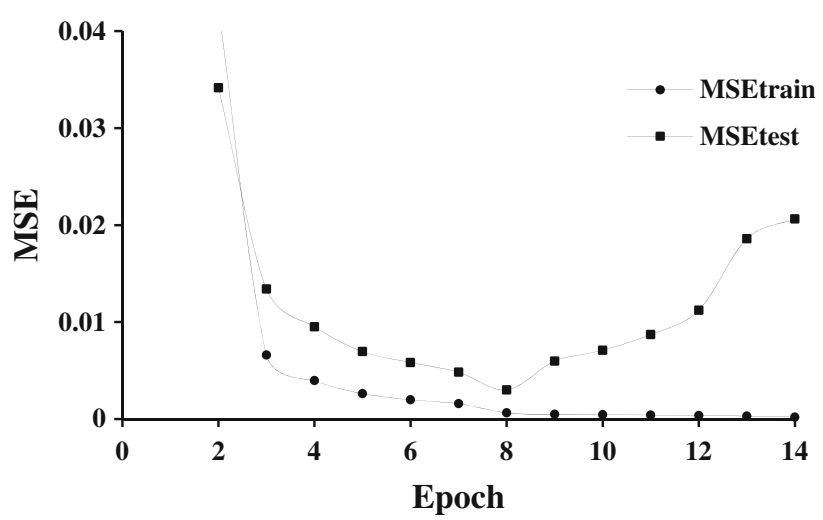

Fig. 6 Optimum number of epoch at the beginning of over-fitting 
iterations MSE of test set increases while MSE of training set decreases or changes slightly. The construction of optimized ANN model is summarized in Table 1. As shown in Fig. 7, maximum fluorescence intensity was due to the excitation wavelength at $365 \mathrm{~nm}$. The spectrofluorimetric curves corresponding to 50 synthetic mixtures of the considered aflatoxins were obtained in excitation wavelength $365 \mathrm{~nm}$, and emission wavelengths between 400 and $600 \mathrm{~nm}$ by the described procedures (Fig. 8). From the 50 synthetic mixtures, three sets with sizes 25,15 and 10 were randomly selected as training, test and prediction sets, respectively. The prepared mixtures of four aflatoxins were between

Table 1 Architectures of the optimized ANN

\begin{tabular}{ll}
\hline Number of nodes in the input layer & 1 \\
Number of nodes in the hidden layer & 3 \\
Number of nodes in the output layer & 4 \\
Number of epoch in the beginning of over-fitting & 8 \\
Momentum & 0.001 \\
Training function & Trainlm \\
\hline
\end{tabular}

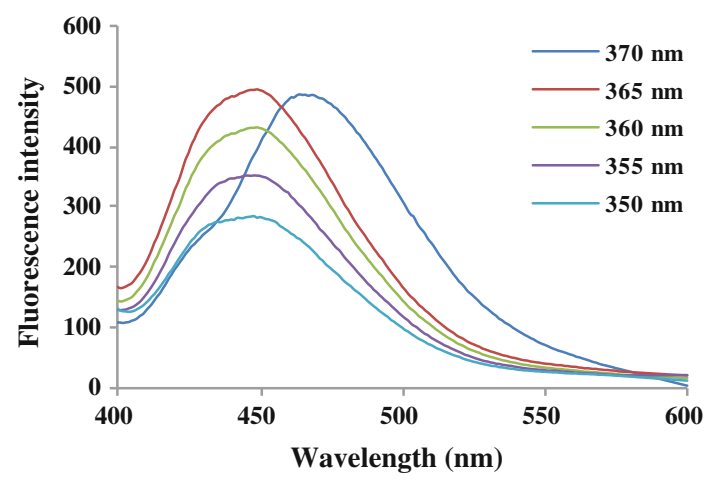

Fig. 7 Fluorescence intensity of aflatoxins in different excitation wavelengths

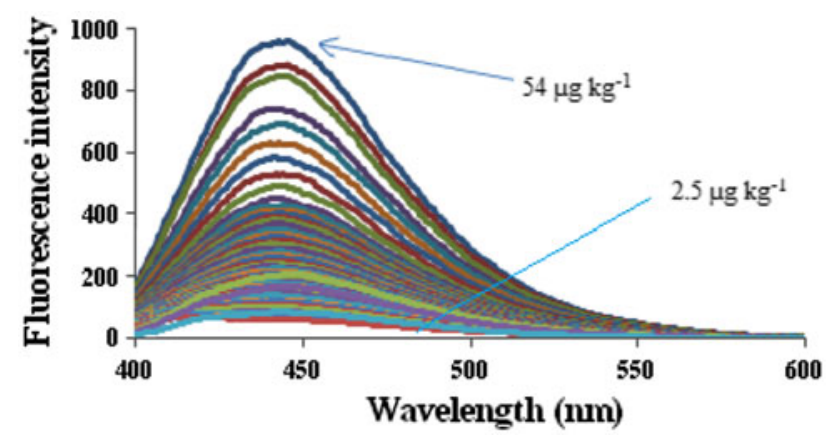

Fig. 8 Fluorescence emission spectra of aflatoxins gold-labeled antibodies with different concentrations (from bottom to top 2.5-54 $\mu \mathrm{g} \mathrm{kg}^{-1}$ ) concentration ranges 0 and $54 \mu \mathrm{g} \mathrm{Kg}^{-1}$. The training and test sets were used for construction and optimization of ANN model, and the independent external prediction set was used to evaluate the quality of the model. The results obtained for test and prediction samples and their statistical parameters are given in Tables 2 and 3, respectively. The reasonable relative errors for each analyte in both sets indicate the accuracy of the proposed method. Performance of ANN for the separation of four aflatoxins was summarized in Fig. 9.

Table 2 Calculated concentrations $\left(\mu \mathrm{g} \mathrm{kg}^{-1}\right.$ ) of aflatoxins by ANN model on test and prediction set

\begin{tabular}{|c|c|c|c|c|c|c|c|c|}
\hline \multirow{2}{*}{$\begin{array}{l}\text { Sample } \\
\text { number }\end{array}$} & \multicolumn{2}{|l|}{ AfB1 } & \multicolumn{2}{|l|}{ AfB2 } & \multicolumn{2}{|l|}{ AfG1 } & \multicolumn{2}{|l|}{ AfG2 } \\
\hline & Actual & Found & Actual & Found & Actual & Found & Actual & Found \\
\hline \multicolumn{9}{|l|}{ Test set } \\
\hline 1 & 0.50 & 0.53 & 0.10 & 0.11 & 0.50 & 0.38 & 0.10 & 0.08 \\
\hline 2 & 0.71 & 0.71 & 0.14 & 0.15 & 0.71 & 0.57 & 0.14 & 0.12 \\
\hline 3 & 1.16 & 1.19 & 0.23 & 0.24 & 1.16 & 1.08 & 0.23 & 0.22 \\
\hline 4 & 1.68 & 1.71 & 0.34 & 0.35 & 1.68 & 1.62 & 0.34 & 0.33 \\
\hline 5 & 2.18 & 2.22 & 0.44 & 0.45 & 2.18 & 2.16 & 0.44 & 0.44 \\
\hline 6 & 2.42 & 2.47 & 0.48 & 0.50 & 2.42 & 2.42 & 0.48 & 0.49 \\
\hline 7 & 2.97 & 3.03 & 0.59 & 0.61 & 2.97 & 3.01 & 0.59 & 0.60 \\
\hline 8 & 3.64 & 3.69 & 0.73 & 0.74 & 3.64 & 3.70 & 0.73 & 0.74 \\
\hline 9 & 4.25 & 4.25 & 0.85 & 0.85 & 4.25 & 4.30 & 0.85 & 0.86 \\
\hline 10 & 5.22 & 5.22 & 1.04 & 1.04 & 5.22 & 5.30 & 1.04 & 1.06 \\
\hline 11 & 6.41 & 6.27 & 1.28 & 1.24 & 6.41 & 6.40 & 1.28 & 1.27 \\
\hline 12 & 7.87 & 7.35 & 1.57 & 1.46 & 7.87 & 7.52 & 1.57 & 1.49 \\
\hline 13 & 8.72 & 8.19 & 1.74 & 1.63 & 8.72 & 8.38 & 1.74 & 1.66 \\
\hline 14 & 13.29 & 14.23 & 2.66 & 2.86 & 13.29 & 14.37 & 2.66 & 2.84 \\
\hline 15 & 20.25 & 19.58 & 4.05 & 3.93 & 20.25 & 19.57 & 4.05 & 3.90 \\
\hline \multicolumn{9}{|c|}{ Prediction set } \\
\hline 1 & 0.98 & 1.07 & 0.20 & 0.22 & 0.98 & 0.95 & 0.20 & 0.20 \\
\hline 2 & 1.36 & 1.37 & 0.27 & 0.28 & 1.36 & 1.26 & 0.27 & 0.26 \\
\hline 3 & 1.97 & 2.01 & 0.39 & 0.40 & 1.97 & 1.94 & 0.39 & 0.39 \\
\hline 4 & 2.68 & 2.75 & 0.54 & 0.55 & 2.68 & 2.72 & 0.54 & 0.55 \\
\hline 5 & 3.29 & 3.36 & 0.66 & 0.67 & 3.29 & 3.36 & 0.66 & 0.67 \\
\hline 6 & 4.71 & 4.74 & 0.94 & 0.94 & 4.71 & 4.80 & 0.94 & 0.96 \\
\hline 7 & 5.78 & 5.64 & 1.16 & 1.12 & 5.78 & 5.75 & 1.16 & 1.15 \\
\hline 8 & 7.10 & 6.78 & 1.42 & 1.35 & 7.10 & 6.93 & 1.42 & 1.38 \\
\hline 9 & 10.76 & 10.58 & 2.15 & 2.11 & 10.76 & 10.78 & 2.15 & 2.14 \\
\hline 10 & 16.40 & 17.39 & 3.28 & 3.51 & 16.40 & 17.48 & 3.28 & 3.45 \\
\hline
\end{tabular}

Table 3 Statistical parameters obtained using simultaneous ANN modeling for total aflatoxins

\begin{tabular}{lllllll}
\hline Aflatoxin & MSET & MSEP & $R_{\text {test }}^{2}$ & $R_{\text {prediction }}^{2}$ & $F_{\text {test }}$ & $F_{\text {prediction }}$ \\
\hline B1 & 0.1283 & 0.1158 & 0.995 & 0.997 & 2,870 & 2,343 \\
B2 & 0.0057 & 0.0063 & 0.995 & 0.995 & 2,617 & 1,751 \\
G1 & 0.1288 & 0.1219 & 0.995 & 0.998 & 2,756 & 4,110 \\
G2 & 0.0047 & 0.0033 & 0.996 & 0.998 & 3,188 & 4,300 \\
\hline
\end{tabular}

$M S E T$ mean square error of test set, MSEP mean square error of prediction set 


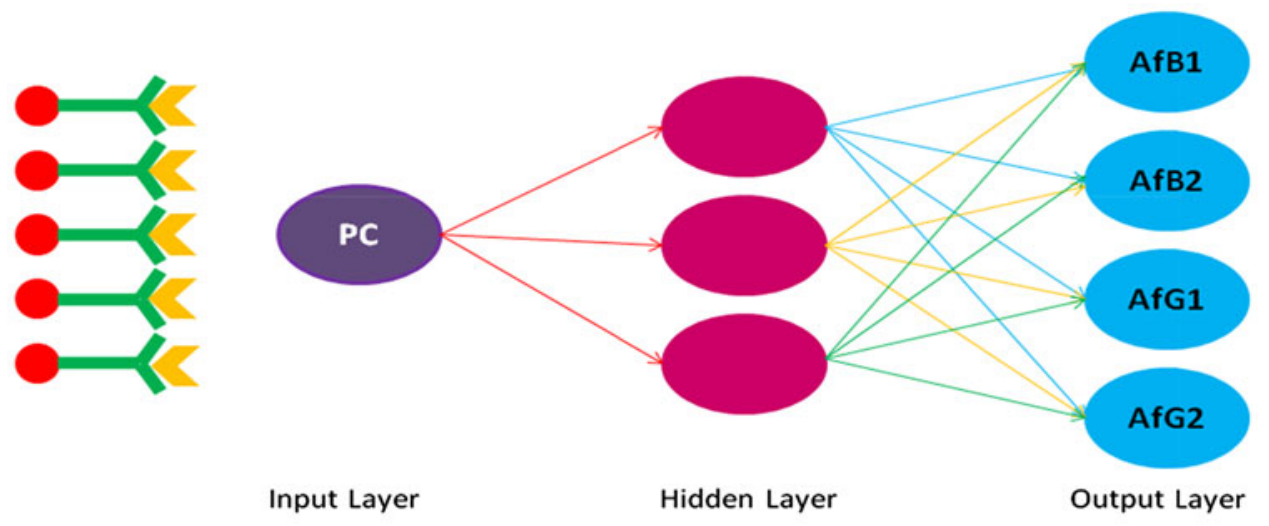

Artificial Neural Network

Gold nanoparticle

Anti-Aflatoxin

Aflatoxin

Fig. 9 Schematic representation of ANN for the separation of four aflatoxins

Table 4 Predicted concentrations $\left(\mu \mathrm{g} \mathrm{kg}^{-1}\right)$ obtained for aflatoxins in some spike samples using ANN method

a Tap water of Shahid Bahonar University of Kerman, Kerman, Iran

b Pistachio of Rafsanjan,

Kerman, Iran

c Mean of three

determinations \pm standard deviation

${ }^{\mathrm{d}}$ Percent average recovery

\begin{tabular}{|c|c|c|c|c|c|}
\hline Reference sample & Aflatoxin & Added Af & HPLC & $\mathrm{ANN}^{\mathrm{c}}$ & $\%$ Recovery $\mathrm{ANN}^{\mathrm{d}}$ \\
\hline \multirow[t]{4}{*}{ Water $^{\mathrm{a}}$} & $\mathrm{B} 1$ & 1.0 & - & $1.020 \pm(0.05)$ & 102.01 \\
\hline & B2 & 0.2 & - & $0.204 \pm(0.01)$ & 101.82 \\
\hline & G1 & 1.0 & - & $1.016 \pm(0.05)$ & 101.62 \\
\hline & $\mathrm{G} 2$ & 0.2 & - & $0.204 \pm(0.01)$ & 101.78 \\
\hline \multirow[t]{4}{*}{ Water $^{\mathrm{a}}$} & $\mathrm{B} 1$ & 3.0 & - & $3.151 \pm(0.02)$ & 105.02 \\
\hline & B2 & 0.6 & - & $0.630 \pm(0.01)$ & 104.98 \\
\hline & G1 & 3.0 & - & $3.149 \pm(0.02)$ & 104.96 \\
\hline & $\mathrm{G} 2$ & 0.6 & - & $0.630 \pm(0.01)$ & 104.94 \\
\hline \multirow[t]{4}{*}{ Pistachio $^{\mathrm{b}}$} & $\mathrm{B} 1$ & 24.0 & 23.3 & $22.6 \pm(0.05)$ & 94.35 \\
\hline & B2 & 6.0 & 5.9 & $5.7 \pm(0.01)$ & 94.33 \\
\hline & G1 & 24.0 & 23.0 & $22.6 \pm(0.06)$ & 94.27 \\
\hline & $\mathrm{G} 2$ & 6.0 & 5.8 & $5.7 \pm(0.01)$ & 94.33 \\
\hline \multirow[t]{4}{*}{ Pistachio $^{\mathrm{b}}$} & $\mathrm{B} 1$ & 16.0 & 15.5 & $15.0 \pm(0.04)$ & 93.73 \\
\hline & B2 & 4.0 & 3.8 & $3.7 \pm(0.01)$ & 93.80 \\
\hline & G1 & 16.0 & 15.7 & $15.0 \pm(0.06)$ & 93.85 \\
\hline & $\mathrm{G} 2$ & 4.0 & 3.8 & $3.7 \pm(0.01)$ & 93.84 \\
\hline \multirow[t]{4}{*}{ Pistachio $^{\mathrm{b}}$} & $\mathrm{B} 1$ & 4.0 & 3.8 & $4.2 \pm(0.05)$ & 105.87 \\
\hline & B2 & 1.0 & 0.98 & $1.06 \pm(0.01)$ & 105.63 \\
\hline & G1 & 4.0 & 3.8 & $4.2 \pm(0.03)$ & 105.30 \\
\hline & G2 & 1.0 & 0.96 & $1.06 \pm(0.01)$ & 105.68 \\
\hline
\end{tabular}

Real sample analysis

For evaluation of the method, water and non-contaminated peanut samples were spiked with four aflatoxins and analyzed. The results were compared with the results obtained using the HPLC method. As shown in Table 4, the proposed method can provide successfully comparable concentration values relative to the routine method of aflatoxin analysis. In addition, this methodology is easier and faster than HPLC, which is normally used to monitor this sort of samples.

Acknowledgments The authors gratefully acknowledge Shahid Bahonar University of Kerman for financial support. 
Open Access This article is distributed under the terms of the Creative Commons Attribution License which permits any use, distribution, and reproduction in any medium, provided the original author(s) and the source are credited.

\section{References}

1. M. Molina, L. Giannuzzi, Food. Res. Int. 35, 585 (2002)

2. M.A. Passone, L.C. Rosso, A. Ciancio, M. Etcheverry, Int. J. Food Microbiol. 138, 276 (2010)

3. N. Sardiñas, C. Vázquez, J. Gil-Serna, M.T. Gónzalez-Jaén, B. Patiño, Int. J. Food Microbiol. 145, 121 (2011)

4. Z. Mayer, A. Bagnara, P. Ferber, R. Geisen, Int. J. Food Microbiol. 82, 143 (2003)

5. M. Schmidt-Heydt, A. Abdel-Hadi, N. Magan, R. Geisen, Int. J. Food Microbiol. 135, 231 (2009)

6. A. Pittet, Rev. Med. Vet. 149, 479 (1998)

7. H. Aycicek, A. Aksoy, S. Saygi, Food Control 16, 263 (2005)

8. C. Juan, A. Zinedine, J.C. Molto, L. Idrissi, J. Manes, Food Control 19, 849 (2008)

9. A.C. Mak, S.J. Osterfeld, H. Yu, S.H. Wang, R.J. Davis, O.A. Jejelowo, Biosens. Bioelectron. 25, 1635 (2010)

10. C.M. Maragos, Anal. Bioanal. Chem. 395, 1205 (2009)

11. M. Sulyok, R. Krska, R. Schuhmacher, Food Chem. 119, 408 (2010)

12. N.W. Turner, S. Subrahmanyam, S.A. Piletsky, Anal. Chim. Acta 632, 168 (2009)

13. P.W. Li, Q. Zhang, W. Zhang, Trends Anal. Chem. 28, 1115 (2009)

14. I.V. Gachok, A.P. Bondarenko, S. De Saeger, M. Lobeau, C. Van Peteghem, W.B. Shim, Toxicol. Lett. 180, S28 (2008)

15. J. Zhang, J. Wang, J. Zhu, J. Xu, H. Chen, D. Xu, Microchim. Acta 163, 63 (2008)

16. X. Zhao, L.R. Hilliard, S. John Mechery, Y. Wang, R.P. Bagwe, S. Jin, PNAS 101, 15027 (2004)

17. M. Oda, M. Morita, H. Unno, Y. Tanji, Appl. Environ. Microbiol. 70, 527 (2004)

18. C. Si, H. Kun-Lun, X. Wen-Tao, L. Yuan, L. Yun-Bo, J. Agric. Biotechol. 4, 15 (2007)

19. N.C. Tansil, Z. Gao, Nano Today 1, 28 (2006)
20. Y. Bai, H. Yang, W. Yang, Y. Li, C. Sun, Sens. Actuators B 124, 179 (2007)

21. P. Pandey, S.P. Singh, S.K. Arya, A. Sharma, M. Datta, B.D. Malhotra, J. Nanosci. Nanotechnol. 8, 3158 (2008)

22. S. Paek, S. Lee, H. Cho, Y. Kim, Methods 22, 53 (2000)

23. S. Xiulan, Z. Xiaolian, T. Jian, J. Zhou, F.S. Chu, Int. J. Food Microbiol. 99, 185 (2005)

24. I. Sato, K. Kojima, T. Yamasaki, K. Yoshida, M. Yoshiike, S. Takano, T. Mukai, T. Iwamoto, Methods 287, 137 (2004)

25. D.J. Chiao, R.H. Shyu, C.S. Hu, H.Y. Chiang, S.S. Tang, J. Chromatogr. B 809, 37 (2004)

26. W.P. Faulk, G.M. Taylor, Immunochemistry 8, 1081 (1971)

27. O. Abbas, C. Rebufa, N. Dupuy, A. Permanyer, J. Kister, Fuel 98, 5 (2012)

28. K.M.G. Limaa, I.M. Raimundo, M.F. Pimentel, Sens. Actuators B 160, 691 (2011)

29. R.M. Balabin, E.I. Lomakina, R.Z. Safieva, Fuel 90, 2007 (2011)

30. A. Safavi, H. Abdollahi, M.R. Hormozi-Nezhad, Talanta 59, 515 (2003)

31. F. Marini, Anal. Chim. Acta 635, 121 (2009)

32. S. Haykin, Neural Networks and Learning Machines (Pearson Education Inc., New Jersey, 2009), pp. 1-60, 122-218

33. J. Tashkhouriana, M.R. Hormozi-Nezhad, J. Khodaveisid, Spectrochim. Acta Part A 82, 25 (2011)

34. S. Ren, L. Gao, Chemom. Intell. Lab. Syst. 107, 276 (2011)

35. G.T. Hermanson, A.K. Mallia, P.K. Smith, Immobilized affinity ligand techniques (Academic Press, San Diego, 1992)

36. M. Vosough, M. Bayat, A. Salemi, Anal. Chim. Acta 663, 11 (2010)

37. M.H. Fatemi, J. Chromatogr. A 955, 273 (2002)

38. P. Korytár, J. Covaci Boer, A. Gelbin, UATh Brinkman, J. Chromatogr. A 1065, 239 (2005)

39. M. Jalali-Heravi, E. Noroozian, M. Mousavi, J. Chromatogr. A 1023, 247 (2004)

40. M. Jalali-Heravi, Z. Garakani-Nejad, J. Chromatogr. A 927, 211 (2001)

41. I.T. Jolliffe, Principal component analysis, 2nd edn. (Springer, New York, 2002)

42. C.N. Rossi, C.R. Takabayashi, M.A. Ono, G.H. Saito, E.N. Itano, O. Kawamura, E.Y. Hirooka, E.Y.S. Ono, Food Chem. 132, 2211 (2012)

43. B. Johne, K. Hansen, E. Mark, J. Holtlund, Methods 183, 167 (1995) 